\title{
Molecular characterization and expression analysis of a suite of cytochrome P450 enzymes implicated in insect hydrocarbon degradation in the entomopathogenic fungus Beauveria bassiana
}

Correspondence

Nemat O. Keyhani

keyhani@ufl.edu

Received 12 March 2010

Revised 14 April 2010

Accepted 21 April 2010

\author{
Nicolás Pedrini, ${ }^{1,2}$ Shizhu Zhang, ${ }^{1}$ M. Patricia Juárez ${ }^{2}$ \\ and Nemat O. Keyhani ${ }^{1}$
${ }^{1}$ Department of Microbiology and Cell Science, University of Florida, Gainesville, FL 32611, USA
${ }^{2}$ Instituto de Investigaciones Bioquímicas de La Plata, CONICET, Facultad de Ciencias Médicas, UNLP, Calles 60 y 120 (1900), La Plata, Argentina

\begin{abstract}
The insect epicuticle or waxy layer comprises a heterogeneous mixture of lipids that include abundant levels of long-chain alkanes, alkenes, wax esters and fatty acids. This structure represents the first barrier against microbial attack and for broad-host-range insect pathogens, such as Beauveria bassiana, it is the initial interface mediating the host-pathogen interaction, since these organisms do not require any specialized mode of entry and infect target hosts via the cuticle. $B$. bassiana is able to grow on straight chain alkanes up to $n-C_{33}$ as a sole source of carbon and energy. The cDNA and genomic sequences, including putative regulatory elements, for eight cytochrome P450 enzymes, postulated to be involved in alkane and insect epicuticle degradation, were isolated and characterized. Expression studies using a range of alkanes as well as an insect-derived epicuticular extract from the blood-sucking bug Triatomas infestans revealed a differential expression pattern for the P450 genes examined, and suggest that $B$. bassiana contains a series of hydrocarbon-assimilating enzymes with overlapping specificity in order to target the surface lipids of insect hosts. Phylogenetic analysis of the translated ORFs of the sequences revealed that the enzyme which displayed the highest levels of induction on both alkanes and the insect epicuticular extract represents the founding member of a new cytochrome P450 family, with three of the other sequences assigned as the first members of new P450 subfamilies. The remaining four proteins clustered with known P450 families whose members include alkane monooxygenases.
\end{abstract}

\section{INTRODUCTION}

Unlike other insect-pathogenic micro-organisms which must be ingested to initiate disease (virus, bacteria, nematodes and protozoa), entomopathogenic fungi, such as Metarhizium anisopliae and Beauveria bassiana, mostly invade target hosts by penetrating through their cuticle.

Abbreviations: AhR, aryl hydrocarbon receptor; ARNT, AhR nuclear translocator; CM, complete medium; MM, minimal medium; PDA, potato dextrose agar; STRE, stress response element; TiLip, lipids extracted from Triatoma infestans.

The GenBank/EMBL/DDBJ accession number for the predicted protein products of the $B$. bassiana cytochrome $P 450$ genes are p450-1, GU566074; p450-2, AM409327; p450-3, GU566075; p450-4, GU566076; p450-5, GU566077; p450-6, GU566078; p450-7, GU566079; and p450-8, GU566080.

A supplementary table of primer sequences and two supplementary figures, showing $B$. bassiana cytochrome P450 gene sequences and amino acid alignments, are available with the online version of this paper.
The outermost insect surface or epicuticle is covered by a lipid-rich layer, usually composed of very long-chain hydrocarbons together with variable amounts of fatty alcohols, fatty acids and wax esters. The epicuticle protects the insect from desiccation, chemical and biological attack, and acts as a platform for host semiochemicals (Blomquist et al., 1987; Figueiras et al., 2009; Juarez, 1994). Entomopathogenic fungi have the ability to degrade insect cuticular lipids with hydrocarbons, the preferred components capable of supporting fungal growth (Napolitano \& Juarez, 1997). The first evidence of the complete catabolism of insect-like hydrocarbons by entomopathogenic fungi was obtained from B. bassiana and M. anisopliae (Crespo et al., 2000). In addition, alkane-grown B. bassiana showed enhanced virulence, by both increasing mortality, i.e. a greater proportion of target hosts killed over time (Crespo et al., 2002), and reducing the time to kill its insect hosts (Pedrini et al., 2009). Although several aspects of the biochemistry of the interaction between entomopathogenic 
fungi and insect host cuticular hydrocarbons have been examined (Pedrini et al., 2007), there is no information concerning the enzymes involved in the initial steps of alkane hydrocarbon degradation. In yeasts, the first oxidation round of the hydrocarbon substrate is catalysed by a microsomal cytochrome P450 enzyme system (Tanaka \& Fukui, 1989). In some instances, besides producing a fatty alcohol as the primary oxidation product, a single cytochrome P450 form has also been shown to catalyse a cascade of monooxidation products (Scheller et al., 1998). Subsequently, the fatty alcohol - or eventually the fatty acid - provides the appropriate fatty acyl-CoA for complete $\beta$ oxidation of the hydrocarbon substrate in peroxisomes (Tanaka \& Ueda, 1993).

The cytochrome P450 monooxigenases constitute a large superfamily of haem-thiolate proteins widely distributed in different life forms including prokaryotes and lower and higher eukaryotes. Collectively, these enzymes play an important role in the metabolism of both endogenous and xenobiotic compounds, often catalysing conversion of a wide range of lipophilic compounds to more hydrophilic derivatives (Guengerich, 2001). The enzymes specifically involved in terminal hydroxylation of n-alkanes, named P450alk, are expressed from genes belonging to the CYP52 family. These genes have been identified in the yeasts Candida tropicalis (Seghezzi et al., 1991, 1992), C. maltosa (Ohkuma et al., 1995), C. apicola (Lottermoser et al., 1996), Yarrowia lipolytica (Iida et al., 1998) and Debaryomyces hansenii (Yadav \& Loper, 1999).

Based on a previous EST dataset (Cho et al., 2006a, b) and newly constructed libraries, we describe the isolation and characterization of eight cytochrome P450 genes of $B$. bassiana. The molecular characterization of these enzymes resulted in their assignment to one novel cytochrome P450 family and four novel subfamilies. Expression analysis of the genes revealed their differential induction by various long chain alkanes and insect lipids, implying overlapping but discrete functions for each enzyme. The potential role of these enzymes in insect hydrocarbon degradation is discussed.

\section{METHODS}

Cultivation of fungi. B. bassiana (ATCC 90517) was routinely grown on potato dextrose agar (PDA). Plates were incubated at $26{ }^{\circ} \mathrm{C}$ for 10-14 days and aerial conidia were harvested by flooding the plate with sterile distilled water. Conidial suspensions were filtered through a single layer of Miracloth and final spore concentrations were determined by direct count using a haemocytometer. For expression studies, fungal cells were grown in complete liquid medium (CM) containing ( $\left(^{-1}\right.$ distilled water) $0.4 \mathrm{~g} \mathrm{KH}_{2} \mathrm{PO}_{4}, 1.4 \mathrm{~g} \mathrm{Na}_{2} \mathrm{HPO}_{4}, 0.6 \mathrm{~g}$ $\mathrm{MgSO}_{4} .7 \mathrm{H}_{2} \mathrm{O}, 1.0 \mathrm{~g} \mathrm{KCl}, 0.7 \mathrm{~g} \mathrm{NH}_{4} \mathrm{NO}_{3} .7 \mathrm{H}_{2} \mathrm{O}, 10 \mathrm{~g}$ glucose and $5 \mathrm{~g}$ yeast extract. Fungi were also cultivated in minimal medium (MM), composed of $\mathrm{CM}$ without the glucose and yeast extract, and supplemented with synthetic hydrocarbons $(1 \% \mathrm{w} / \mathrm{v})$ as follows: $\mathrm{n}-$ hexadecane $\left(n-C_{16}\right)$, n-eicosane $\left(n-C_{20}\right)$, n-tetracosane $\left(n-C_{24}\right)$ or $n$ octacosane $\left(\mathrm{n}-\mathrm{C}_{28}\right)$. MM was also added with cuticular lipids extracted from the blood-sucking bug Triatoma infestans (TiLip).
The extract was prepared by submerging 600 fifth instar exuviae in hexane $\left[6 \mathrm{ml}\right.$ hexane $\left.(\mathrm{g} \text { exuviae })^{-1}\right]$ for $48 \mathrm{~h}$. The extract was transferred to another vial, and reduced in volume under nitrogen. Regardless of the carbon source provided, the fungi grew on the surface of the biphasic incubation media. Flasks were incubated at $26{ }^{\circ} \mathrm{C}$ for 10 days without aeration.

Fungal growth on alkanes. Fungal spores were harvested from PDA plates directly into sterile distilled water and were washed twice with the same solution; the suspension was then adjusted to $10^{7}-10^{8}$ conidia $\mathrm{ml}^{-1}$ after counting using a haemocytometer. Spore suspensions $(5-10 \mu \mathrm{l})$ were then placed into the middle of microtitre agar plates (24- or 48-well). For 24-well plates, each well contained $1 \mathrm{ml} \mathrm{MM}$ in Noble agar overlaid with the desired alkane $(0.1 \mathrm{ml}$ of a $10 \%$ hydrocarbon stock solution in hexane) as a carbon source.

Confocal microscopy. For observation of fungal growth on alkanes, 5-10 $\mu$ of $10 \%$ hydrocarbon solution was spotted and allowed to air

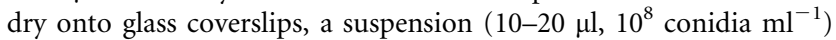
of fungal spores was overlaid on the dried spot and incubated at $26{ }^{\circ} \mathrm{C}$, with high humidity ( $>80 \% \mathrm{RH}$ ), for $24-48 \mathrm{~h}$. Subsequently, a solution containing the fluorescent dye FM4-64 (1 mM stock solution in ethanol; Invitrogen) was added to the coverslip to a final concentration of $2-10 \mu \mathrm{M}$. Samples were incubated at room temperature for $1 \mathrm{~h}$ prior to visualization. Mounted slides were observed using a Zeiss Axiovert PASCAL LSM5 confocal microscope fitted with Nomarski differential interference contrast optics and an LP560 filter $\left(\mathrm{Ex}_{543} / \mathrm{Em}_{560}\right)$ or LP505 filter $\left(\mathrm{Ex}_{488} / \mathrm{Em}_{518}\right)$ for FM4-64 and CDCFDA visualization, respectively. Images were recorded with a real-time digital imaging set-up and were arranged by using Adobe Photoshop or similar software.

Full-length gene sequence isolation. P450 gene fragments with high homology to P450alk were identified from previously obtained EST libraries (Cho et al., 2006a, b). To obtain full-length cDNA sequences for cytochrome p450-1 to -7 , fungal cells were ground in liquid nitrogen and total RNA was extracted using an RNAeasy plant mini kit (Qiagen). Several aliquots of total RNA isolated from several samples were pooled in order to construct a representative cDNA library. Full-length sequences were isolated by $5^{\prime}$ - and $3^{\prime}$-rapid amplification of cDNA ends (Clontech). Amplified products were cloned into pGEM-T Easy vector system (Promega) and transformed into JM-109 cells. The presence of inserts was detected using the blue/ white screening on X-Gal/LB agar plates. The gene-specific primers used are shown in Supplementary Table S1 (available with the online version of this paper). The full-length cDNA sequence for the $B$. bassiana cytochrome $p 450-8$ and the genomic DNA sequences for all of the P450s were obtained from Drs S. H. Ying and M. G. Feng, Institute of Microbiology, Zhejiang University, Hangzhou, China ( $B$. bassiana whole genome sequencing, in progress).

Sequence analyses. The sequences were analysed using programs implemented at the Biology WorkBench website (http://workbench. sdsc.edu/); splice site analysis was performed using the FSPLICE program at the SoftBerry server (http://www.softberry.com/berry. phtml). Introns were detected by visual comparison to both cDNA and DNA sequences, in zones where the former were available, by either EST or full-length cDNA. Coding sequences were translated to amino acid sequences and were aligned using the ExPASy tools (ExPASy Proteomics Server). Phylogenetic analysis was performed with the Phylogeny.fr platform (Dereeper et al., 2008), which uses the programs MUSCLE for multiple alignment, Gblocks for automatic alignment curation, PhyML for tree building and TreeDyn for tree drawing.

The sequences obtained were submitted both to GenBank for gene annotation and to David Nelson (University of Tennessee, Memphis, 
USA) for assignment of the official cytochrome P450 names, based on criteria established by the P450 Nomenclature Committee (http:// drnelson.uthsc.edu/CytochromeP450.html).

Gene expression analysis. Total RNA was obtained as described above, which included an on-column DNA digestion step. RNA was quantified by using the RiboGreen RNA quantification kit (Molecular Probes), and the integrity was assessed on a $1 \%(\mathrm{w} / \mathrm{v})$ agarose gel. Twostep RT real-time PCR was carried out with the iScript cDNA synthesis kit and iQ SYBR Green supermix (Bio-Rad). The primers used are shown in Supplementary Table S1. Amplification was performed in an iCycler Thermal Cycler (Bio-Rad), employing 20 ng reverse-transcribed total RNA for each sample $\left(C M, n-C_{16}, n-C_{20}, n-C_{24}, n-C_{28}\right.$ or TiLip). The following amplification programme was used: denaturation at $95{ }^{\circ} \mathrm{C}$ for $10 \mathrm{~min}$, followed by 40 cycles with three-segment amplification $\left(30 \mathrm{~s}\right.$ at $95{ }^{\circ} \mathrm{C}$ for denaturation, $30 \mathrm{~s}$ at $64{ }^{\circ} \mathrm{C}$ for annealing and $30 \mathrm{~s}$ at $72{ }^{\circ} \mathrm{C}$ for polymerase elongation). In order to confirm that only single products were amplified, a temperature melting step was then performed. The calibration curve method was used for the analysis of data obtained from the RT-PCR system, with ribosomal 5.8S RNA as the housekeeping gene. Negative controls were performed by using 'cDNA' generated without reverse transcriptase as templates. Reactions containing primer pairs without template were also included as blank controls. The assay was done in duplicate for each of the three independent biological replicates performed.

\section{RESULTS}

\section{Fungal growth on alkanes}

Although $B$. bassiana is capable of growing on insect cuticular hydrocarbons, the chain length range that could support growth of the fungus as the sole source of carbon and energy is not known. Therefore, the growth of $B$. bassiana on a range of aliphatic alkanes, including $\mathrm{n}-\mathrm{C}_{9-24}$,
$\mathrm{n}-\mathrm{C}_{26-28}, \mathrm{n}-\mathrm{C}_{30,31,33,35,39,41}$, in both liquid and solid media conditions was tested (Fig. 1a). B. bassiana was able to grow on all alkanes tested as the sole carbon source except for the three longest chain alkanes, $\mathrm{n}-\mathrm{C}_{35}, 39,41$, with the mid-range alkanes (around $n-C_{16}$ ) appearing to be the preferred alkane substrate for growth as a sole carbon source. Some growth on the three longest chain alkane substrates was noted when spores harvested from $n-C_{20^{-}}$ grown cells were used as the inocula, indicating that higher chain alkanes may be degraded under specific conditions, or growth on these substrates may be an adaptive response. Directed and higher density hyphal growth towards the alkane nutrient source could also be visualized in experiments in which fungal conidia were overlaid and/or placed near the alkane substrate source (Fig. 1b). Attempts at visualizing potential hydrocarbon transport by incubation of the fungal cells growing on alkanes $\left(n-C_{16}\right)$ with the lipophilic dye FM4-64 resulted in bright membrane staining; however, no internal lipid bodies could be detected under the conditions used. Similar directed and higher density growth was seen in experiments using $\mathrm{C}_{20}$, $\mathrm{C}_{22}$ and $\mathrm{C}_{28}$, as well as with TiLip.

\section{Full-length sequences and nomenclature}

A suite of cytochrome P450 gene fragments ( $p 450-1$ to p450-8) was identified in a set of EST libraries, with sequence lengths varying from 207 to $736 \mathrm{bp}$. Full-length cDNA genomic sequences were obtained as described in Methods. The gene p450-2 was coincident with one of the genes belonging to a previously characterized contig of 21932 bp (GenBank accession no. AM409327), which is

(b)

(a)
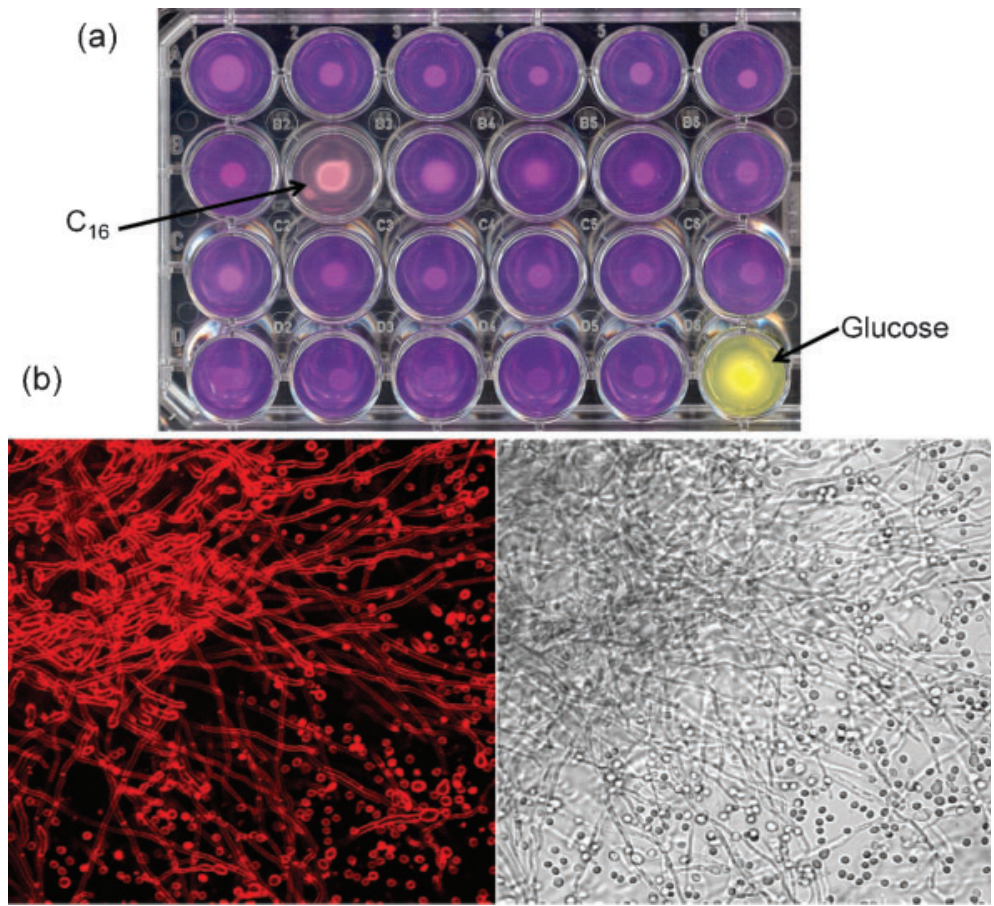

Fig. 1. (a) Growth of B. bassiana inoculated in the centre of microtitre wells containing $\mathrm{MM}$, bromocresol purple $(0.05 \%)$ and the indicated n-alkane $(0.1 \% \mathrm{w} / \mathrm{w})$ as the sole carbon source. Top row, $\mathrm{C}_{9}-\mathrm{C}_{14}$; row 2, $\mathrm{C}_{15}-\mathrm{C}_{20}$; row $3, \mathrm{C}_{21}-\mathrm{C}_{24}, \mathrm{C}_{26}, \mathrm{C}_{27}$; row $4, \mathrm{C}_{28}-\mathrm{C}_{31}$, $\mathrm{C}_{33}$, glucose. (b) Differential interference contrast and fluorescence images of FM4-64 stained fungal cells growing on $\mathrm{C}_{16}$. The droplet containing the alkane is in the upper left-hand corner of the image. 
involved in the biosynthesis of the 2-pyridone tenellin in $B$. bassiana (Eley et al., 2007).

Based on the analysis of the predicted protein products of the $B$. bassiana cytochrome $\mathrm{P} 450$ gene, according to the established criteria of the P450 Nomenclature Committee, p450-3 was found to represent the first member of a novel cytochrome P450 family, whereas P450-1, p450-6 and p450-8 were assigned as the first genes belonging to three novel subfamilies. The official names assigned were CYP52X1 ( $p 450-1$, GenBank accession no. GU566074), CYP655C1 (p450-2, AM409327), CYP5337A1 (p450-3, GU566075), CYP52G11 ( $p 450-4$, GU566076), CYP539B5 ( $p 450-5$, GU566077), CYP617N1 (p450-6, GU566078), CYP53A26 (p450-7, GU566079) and CYP584Q1 (p450-8, GU566080).

\section{Sequence analysis}

Other than in p450-7, for which not enough $5^{\prime}$ flanking sequence was isolated, a variety of genetic elements and repeated sequences were found in the upstream region of the genes (Fig. 2 and Supplementary Fig. S1, available with the online version of this paper). Among these, a pentanucleotide repeat element ATGTG (or its complement CACAT) appeared in $p 450-2$ at position -939, twice in $p 450-1$ (beginning at positions -613 and -1033), p450-5 (-413 and -991), p450-6 (-561 and $-864)$ and $p 450-8(-29$ and -266$)$, and three times in p450-3 (-19, -322 and -434). These sequences have been previously reported in promoters of P450alk (alkanespecific) genes of the yeast C. maltosa (Ohkuma et al.,
1995) and D. hansenii (Yadav \& Loper, 1999). A TATA box was present in the putative promoters beginning at -152 (p450-1), -216 (p450-2), -277 (p450-3), - 131 (p450-4), -374 (p450-5), -57 ( $p 450-6)$ and -131 (p450-8). Also, CCAAT box sequences were detected at positions -438 $(p 450-1),-353,-381$ and -509 (p450-2), $-88,-377$, -745 and -874 ( $p 450-4),-562$ ( $p 450-5)$ and -49 (p450-6). Several sequences belonging to the core binding motif of the xenobiotic responsive element, which participates in the induction of CYP genes via interactions with the aryl hydrocarbon receptor (AhR) and the AhR nuclear translocator (ARNT) were identified (Swanson, 2002; Tsuchiya et al., 2003). The sequences CACGC or CANNTG, which are potentially recognized by the AhR/ARNT heterodimer and/ or the corresponding homodimers, were found at positions $-352,-432,-687,-794,-935,-982$ and -1035 (p450-1), -105, -299, -351, -360, -427, -862, - 1005 and -1173 ( $p 450-2),-200,-322$ and -437 (p450-3), $-428,-1416$ and $-1460(p 450-4),-412,-633,-773$ and -881 ( $p 450-5),-444,-517,-561,-657,-846,-865$, -1061 and -1107 (p450-6), and $-78,-150$ and -408 (p450-8). Also, the pentanucleotide CCCCT (or its complement), corresponding to the stress response element (STRE), was found in all the genes. This sequence was repeated three times in $p 450-1$ (at positions $-235,-505$ and -540), once in p450-2 (-554), p450-4 (-799), p450-6 $(-222)$ and $p 450-8(-206)$, and twice in $p 450-5(-61$ and -893). The STRE has been described in several promoters of Saccharomyces cerevisiae, and it is related to gene induction by xenobiotic agents and environmental and physiological conditions (Treger et al., 1998). This element was also found in promoters for the P450alk gene of the yeast D. hansenii

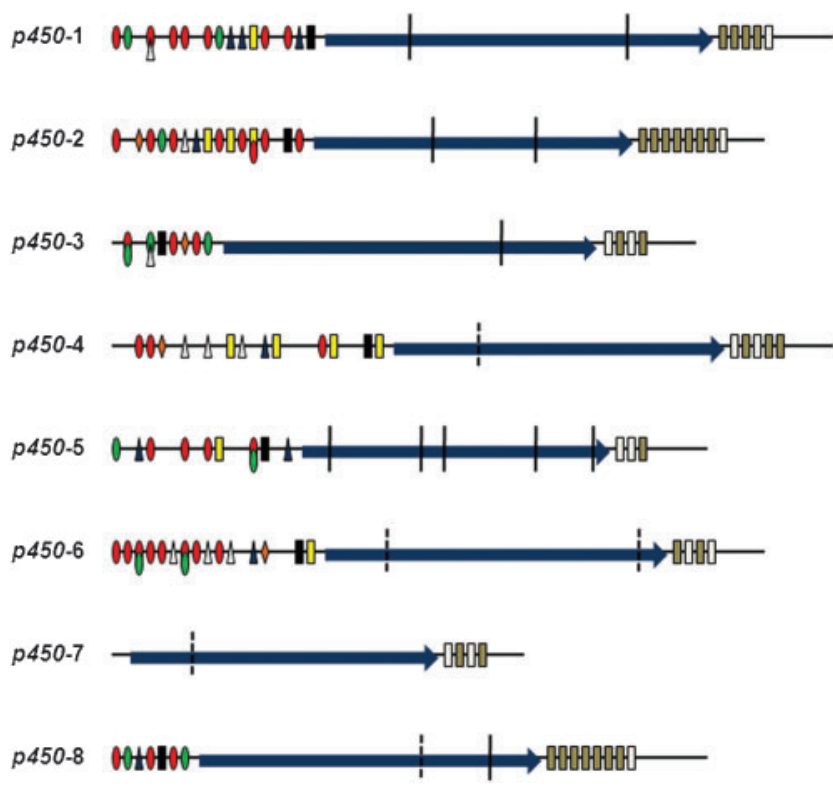

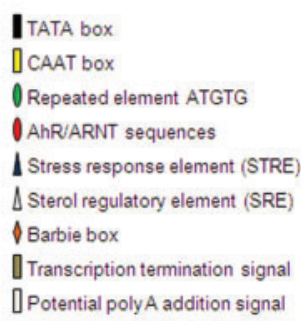

ItATA box

ORepeated element ATGTG

O Ahr/arnt sequences

\Stress response element (STRE)

$\triangle$ Sterol regulatory element (SRE)

Transcription termination signal

DPotential poly A addition signal

Fig. 2. Scheme of $B$. bassiana $P 450$ genes. The ORFs are represented by a blue arrow. Vertical lines mark positions of predicted (dsshed line) and confirmed (solid line) introns. Genetic elements and repeated sequences in both $5^{\prime}$ and $3^{\prime}$ noncoding regions are shown in different colours. 
(Yadav \& Loper, 1999), but as far as we know, it was not previously described in $\mathrm{P} 450$ promoters of filamentous fungi. Other putative regulatory sequences (Barbie box, sterol regulatory elements) detected in the $5^{\prime}$ upstream region of some genes are shown in Fig. 2 and Supplementary Fig. S1. The region surrounding the start codon contains a purine $(G$ or A) at the -3 position, as originally reported for 18 highly expressed genes in S. cerevisiae (Hamilton et al., 1987), and later in fungal CYP genes (Iida et al., 1998; Ohkuma et al., 1995; Seghezzi et al., 1991; Yadav \& Loper, 1999; Yadav et al., 2003). The $3^{\prime}$ non-coding region of all the genes was characterized by the presence of a polyadenylation formation signal (Fig. 2). Only $p 450-7$ exhibited the common conserved AATAAA-3' sequence, whereas the other P450 genes showed different hexameric variants (Graber et al., 1999; Shen et al., 2008) (Supplementary Fig. S1), confirming that this signal is less conserved in fungi (Shen et al., 2008). Variants of the tripartite signal [5'-TAG...TA(T/A)GT...TTT], which indicate potential transcription termination, were also found in the $3^{\prime}$ flanking region (Supplementary Fig. S1). A local alignment between the $5^{\prime}$ sequences available for the examined P450 genes found no obvious novel common sequence element.

All the genes analysed contain at least one intron (Fig. 2, Supplementary Fig. S1). p450-1, p450-2, p450-6 and p450-8 have two introns each; sizes were 109 and 167 bp (p450-1), 62 and $64 \mathrm{bp}(p 450-2), 65$ and $56 \mathrm{bp}(p 450-6)$, and 68 and 69 bp (p450-8). p450-3, p450-4 and p450-7 contain one intron each with lengths of 77, 61 and $66 \mathrm{bp}$, respectively. p450-5 has five small introns of $47,49,54,57$ and $78 \mathrm{bp}$. All the exon/intron boundaries conformed to the canonical GT/AG donor/acceptor rule.

\section{Deduced proteins}

Based upon the isolated cDNA sequences as well as conceptual translation of the genomic DNA sequences using predicted splicing of identified introns, ORFs with typical lengths and domain structures of cytochrome P450s were reconstructed (Supplementary Fig. S2). The length, estimated molecular mass and isoelectric point for each enzyme are shown in Table 1. All the deduced proteins had at least one hydrophobic transmembrane segment (ranging from 17 to 24 aa) as predicted by TMpred analysis, indicating the membrane-bound nature of the P450s. The alignment showed a lower identity in the $\mathrm{N}$-terminal region than in the distal extreme. The three conserved regions helix-I, the site for alkane-binding (Sanglard \& Loper, 1989; Yadav \& Loper, 1999), helix-K and HR-2 (haem-binding) are boxed in Supplementary Fig. S2.

\section{Phylogenetic analysis}

A phylogenetic analysis of the eight P450 deduced amino acid sequences isolated from B. bassiana was performed using the Phylogeny.fr platform (Dereeper et al., 2008). The tree was constructed to include one member of each
Table 1. Properties of deduced B. bassiana cytochrome P450 monooxygenase proteins

\begin{tabular}{|ccccc|}
\hline Protein & $\begin{array}{c}\text { Length } \\
\text { (aa) }\end{array}$ & $\begin{array}{c}\text { Molecular } \\
\text { mass (kDa) }\end{array}$ & pI & $\begin{array}{c}\text { Transmembrane } \\
\text { segment position* }\end{array}$ \\
\hline P450-1 & 528 & 59.5 & 7.72 & $2-26$ \\
P450-2 & 508 & 58.4 & 8.45 & $8-29$ \\
P450-3 & 534 & 58.3 & 8.82 & $1-20$ \\
P450-4 & 528 & 59.3 & 8.24 & $2-18$ \\
P450-5 & 515 & 58.7 & 6.48 & $10-32$ \\
P450-6 & 552 & 61.0 & 6.45 & $11-29,36-62$ \\
P450-7 & 513 & 57.6 & 7.66 & $6-26$ \\
P450-8 & 539 & 61.3 & 8.32 & $20-39$ \\
& & & & \\
\hline
\end{tabular}

${ }^{*}$ Position in no. of amino acids from N-terminus, starting Met.

CYP52 subfamily (P450alk) from yeast, and members of the CYP53 subfamily (benzoate hydroxylase), and the CYP63 subfamily from filamentous fungi. The CYP51 from S. cerevisiae (lanosterol 14-demethylase) was used as reference, since it is the most conserved P450 gene common to all fungi (Aoyama et al., 1996). The B. bassiana sequences separate into two distinct clusters (Fig. 3). The first, consisting of the CYP53 family, included B. bassiana P450-6 and P450-7. The second major cluster included the CYP52 and 63 families; however, only P450-8 clustered within these two families, whereas the rest of the $B$. bassiana $\mathrm{P} 450$ proteins, (P450-1 to -5) subclustered together but appeared distantly related to P450-8.

\section{Cytochrome P450 induction by alkanes and insect lipids}

The expression patterns of the eight $B$. bassiana cytochrome $\mathrm{P} 450$ genes isolated were examined by quantitative PCR. In order to examine whether the P450 genes were specifically induced by alkanes, fungal cultures were grown on n-hexadecane $\left(\mathrm{n}-\mathrm{C}_{16}\right), \mathrm{n}$-eicosane $\left(\mathrm{n}-\mathrm{C}_{20}\right)$, n-tetracosane $\left(n-C_{24}\right)$, or $n$-octacosane $\left(n-C_{28}\right)$ as the sole carbon source and the expression patterns of the $\mathrm{P} 450$ genes were compared with cells grown on CM (glucose) (Fig. 4). In all instances, the expression pattern of ribosomal 5.8S RNA was used as an internal control. The expression of $B$. bassiana p450-1 was slightly induced by growth on the shorter chain hydrocarbons $\left(\mathrm{n}-\mathrm{C}_{16}\right.$ and $\left.\mathrm{n}-\mathrm{C}_{20}\right)$, displayed $\sim 10$-fold induction by the intermediate chain length alkane $\left(\mathrm{n}-\mathrm{C}_{24}\right)$, but was induced almost 100 -fold by the very long chain alkane $\left(n-C_{28}\right)$. In contrast, p450-2, p450-3, p450-6 and $p 450-7$ expression was greatly induced by all alkanes tested (at least more than 50-fold), with p450-3 showing the largest fold induction (300-600-fold) of the genes examined when B. bassiana was grown on all of the alkane substrates tested. Induction of p450-4 expression was nominal under the conditions tested but appeared somewhat enhanced during growth on the highest alkane chain lengths tested (three- to fourfold after growth on n$\mathrm{C}_{24}$ and $\mathrm{n}-\mathrm{C}_{28}$ ). In contrast with the other $\mathrm{P} 450$ genes 


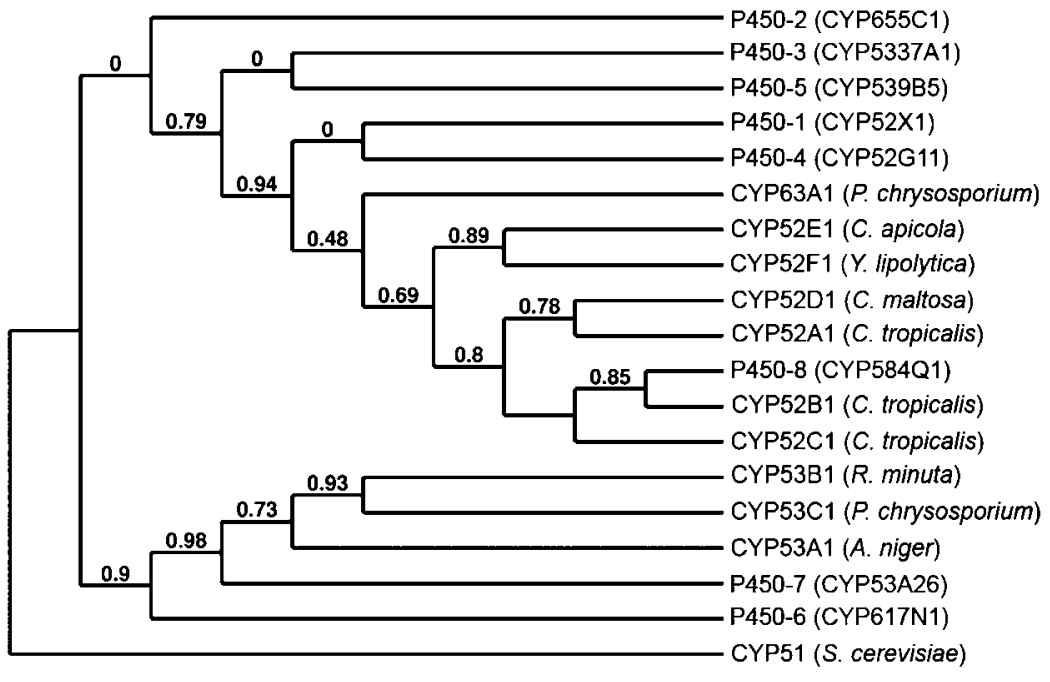

Fig. 3. Phylogenetic tree of the $B$. bassiana P450 genes. The analysis was based on deduced amino acid sequences of P450 genes with known function from different species of yeast and filamentous fungi. Phylogenetic analysis was performed with the Phylogeny.fr platform as described in Methods. Numbers at nodes indicate bootstrap values. CYP51 (S. cerevisiae) was used as reference since it has the most conserved P450 gene that is common to all fungi. examined, induction of $p 450-5$ did not seem to follow a trend; cells grown on $n-C_{20}$ and $n-C_{28}$ were induced $\sim 10$ fold and slightly lower induction was observed when the cells were grown on $n-C_{16}$ and $n-C_{24}$ (two- to fourfold), although overall induction remained low in the presence of the alkanes tested compared with some of the other P450 genes. Little to no induction of $p 450-8$ was observed in any of the alkane growth conditions examined.

In order to examine the expression of the P450 genes induced by insect-derived lipids, $B$. bassiana was grown on a cuticular lipid extract obtained from the blood-sucking bug $T$. infestans, as described in Methods. Three distinct induction profiles were noted: highly induced $B$. bassiana P450 genes ( $>200$ fold), including $p 450-2$ and $p 450-6$; genes moderately induced ( $>30$-fold by the insect extract), including p450-1, p450-3 and p450-7; and genes that displayed low to no induction, p450-4 (fourfold), p450-5 (12-fold) and p450-8 (no induction) (Fig. 5). These values were similar to those obtained with the synthetic alkanes, although p450-3 induction was noticeably higher when grown on the alkanes than on the insect-derived lipid, whereas the insect lipid was the best inducer for p450-5.

\section{DISCUSSION}

The entomopathogenic fungus $B$. bassiana belongs to a group of broad-host-range insect pathogens that do not require any specialized route of infection. Fungal infection of hosts begins with cuticular attachment and degradation, followed by penetration and proliferation within the insect body (Holder \& Keyhani, 2005; Holder et al., 2007; Wanchoo et al., 2009). Although interaction with the outer insect waxy layer (epicuticle) is the first step in pathogenesis, the fungal genes that underlie insect waxy layer degradation remain almost completely unexplored. Our results show that B. bassiana is capable of growing on straight chain alkanes up to $n-C_{33}$, as the sole source of carbon and energy. The lipophilic dye FM4-64 has been previously used to examine nutrient uptake in $B$. bassiana (Lewis et al., 2009), and here we have used it to show

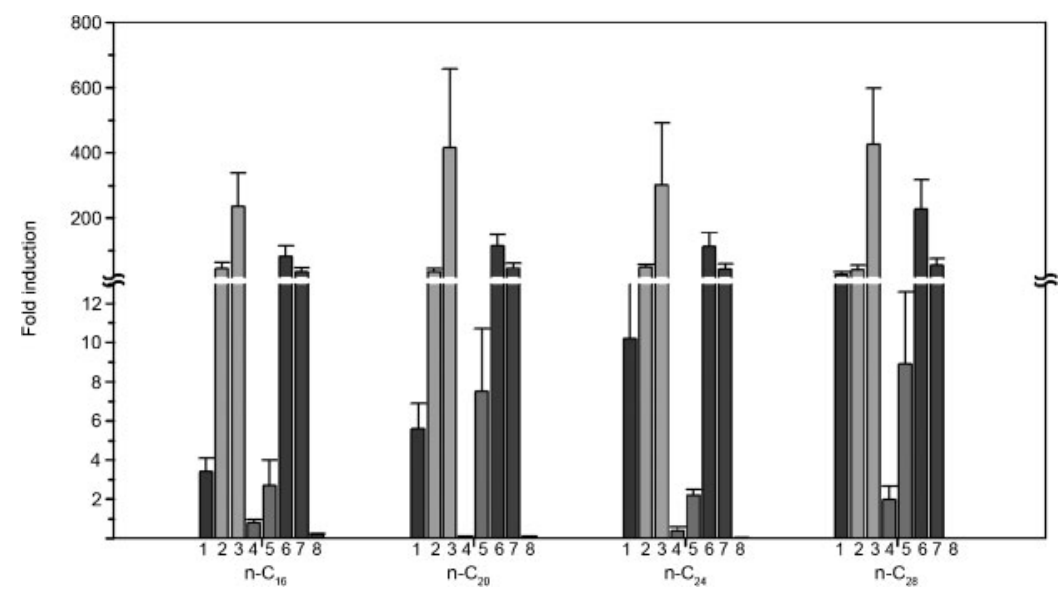

Fig. 4. B. bassiana cytochrome $\mathrm{P} 450$ gene expression analysis as determined by qRTPCR during growth on various alkanes normalized to expression during growth on glucosecontaining media as described in the text. Columns indicate analysis of $p 450-1$ to -8 . Values are means \pm SEM. 


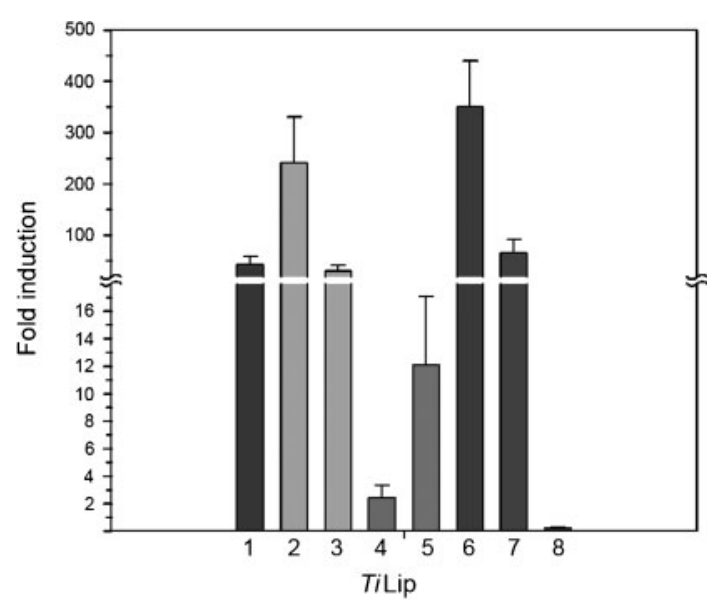

Fig. 5. Expression analysis of $B$. bassiana cytochrome $P 450$ during growth on TiLip. Columns indicate analysis of $p 450-1$ to -8 . Values are means \pm SEM.

directed growth of $B$. bassiana hyphae towards the alkane source.

Cytochrome P450s constitute a superfamily of haemthiolate monooxygenases that act on a wide variety of endogenous and xenobiotic molecules, of which alkanes, a major hydrocarbon component of many insect cuticles, represent a subset of the substrates for these enzymes (Montellano, 2005). The pathway for alkane oxidation in eukaryotic micro-organisms begins with a cytochrome P450 enzyme system. A P450 monooxygenase (P450alk or alkane hydroxylase), acting together with an NADPH cytochrome P450 reductase, was initially described in a number of yeasts, with their distribution in filamentous fungi being scarce (Tanaka \& Fukui, 1989; van Beilen et al., 2003). Our results, the first regarding P450alk genes in entomopathogenic fungi, indicate that $B$. bassiana contains at least seven or eight P450alk genes, corresponding to seven different families. The expression pattern of the enzymes was examined using long chain alkanes as well as epicuticular lipids extracted from $T$. infestans. This latter growth substrate was composed of $37 \%$ hydrocarbons, $29 \%$ triacylglycerols, $15 \%$ fatty alcohols, $8 \%$ free fatty acids and $7 \%$ waxes, and our strain of B. bassiana displays similar levels of virulence to this host, as has been reported for other B. bassiana strains (Juarez \& Fernandez, 2007; Pedrini et al., 2009). Additional cytochrome P450 sequences were also detected in the available EST dataset; however, they were not included in this study because of their low homology with known P450alk sequences.

Other than p450-1, all of the genes contained small introns, in agreement with what has been reported for the CYP63 family of the fungus Phanerochaete chrysosporium (ranging from 35 to $82 \mathrm{bp}$ ) (Yadav et al., 2003). The introns found in p450-1 were similar to those observed for CYP53B1 of Rhodomonas minuta (Fujii et al., 1997) and CYP53C2 in P. chrysosporium (Matsuzaki \& Wariishi, 2005), which ranged from $\sim 50$ to $\sim 250 \mathrm{bp}$. Within a P450 family, the intronexon organization has been proposed to be conserved (Gotoh, 1993); however, this was not the case for all of the B. bassiana genes examined. For example, p450-7 is a member of the CYP53 family, which normally contain multiple introns (CYP53B1 of $R$. minuta has seven), but the $B$. bassiana gene has only one intron. Furthermore, p450-1 has introns similar in length to those observed in the CYP53 family; however, phylogenetically this gene clusters remotely from this family.

B. bassiana p450-3 represents the first member of a new cytochrome P450 family, CYP5337, and it also was the gene whose expression was most highly induced in response to the hydrocarbons tested. In contrast, $B$. bassiana p450-4, classified within the alkane-substrateutilizing CYP52 family, was not highly induced under any of the conditions tested, unlike in alkane-utilizing yeasts (van Beilen et al., 2003). However, p450-1, also a CYP52 family member, was induced by the longer chain alkanes $\mathrm{n}-\mathrm{C}_{24}$ and $\mathrm{n}-\mathrm{C}_{28}$, as well as by the insect epicuticular extract, but was not induced significantly by the shorter chain alkanes tested.

B. bassiana $p 450-2$, $p 450-6$ and $p 450-7$ were induced by all the substrates tested, including the insect-derived extract, reaching $\sim 250-350$-fold induction levels, suggesting an important role for these genes in the first step of insect lipid oxidation. Intriguingly, $p 450-2$ has been previously demonstrated to be involved in tenellin synthesis (with aromatic intermediates) (Halo et al., 2008). Although further experiments are needed, our results might suggest multiple roles (i.e. diverse substrates) for some P450s in different cell processes.

In conclusion, our data indicate that B. bassiana probably contains multiple hydrocarbon-assimilating cytochrome P450 enzymes that may display overlapping substrate specificities. Molecular approaches, including targeted gene knock-outs of specific P450 genes, in order to examine their substrate specificities and confirm their role in insect hydrocarbon degradation, are warranted. Furthermore, these genes would be appropriate potential candidates for overexpression in $B$. bassiana in order to increase its targeting and virulence towards insect pests.

\section{ACKNOWLEDGEMENTS}

The authors wish to thank Urouge Ahktar for technical assistance with some experiments, Drs S. H. Ying and M. G. Feng, Institute of Microbiology, Zhejiang University, Hangzhou, China, for $B$. bassiana genomic information, and to the National Council for Scientific and Technical Research (CONICET) and Fulbright Commission for fellowships for N.P. This work was partially supported by grants of the National Agency for Science and Technology Promotion in Argentina (PICT 2003 01-14174 and 2007 01503), to M.P.J. Both N.P. and M.P.J. are members of the CONICET Researcher's Career. 


\section{REFERENCES}

Aoyama, Y., Noshiro, M., Gotoh, O., Imaoka, S., Funae, Y., Kurosawa, N., Horiuchi, T. \& Yoshida, Y. (1996). Sterol 14demethylase P450 (P45014DM*) is one of the most ancient and conserved P450 species. J Biochem 119, 926-933.

Blomquist, G. J., Nelson, D. R. \& De Renobales, M. (1987). Chemistry, biochemistry, and physiology of insect cuticular lipids. Arch Insect Biochem Physiol 6, 227-265.

Cho, E. M., Boucias, D. \& Keyhani, N. O. (2006a). EST analysis of cDNA libraries from the entomopathogenic fungus Beauveria (Cordyceps) bassiana. II. Fungal cells sporulating on chitin and producing oosporein. Microbiology 152, 2855-2864.

Cho, E. M., Liu, L., Farmerie, W. \& Keyhani, N. O. (2006b). EST analysis of cDNA libraries from the entomopathogenic fungus Beauveria (Cordyceps) bassiana. I. Evidence for stage-specific gene expression in aerial conidia, in vitro blastospores and submerged conidia. Microbiology 152, 2843-2854.

Crespo, R., Juárez, M. P. \& Cafferata, L. F. R. (2000). Biochemical interaction between entomopathogenous fungi and their insect-hostlike hydrocarbons. Mycologia 92, 528-536.

Crespo, R., Juárez, M. P., Dal Bello, G. M., Padin, S., Fernández, G. C. \& Pedrini, N. (2002). Increased mortality of Acanthoscelides obtectus by alkane-grown Beauveria bassiana. BioControl 47, 685-696.

Dereeper, A., Guignon, V., Blanc, G., Audic, S., Buffet, S., Chevenet, F., Dufayard, J. F., Guindon, S., Lefort, V. \& other authors (2008). Phylogeny.fr: robust phylogenetic analysis for the non-specialist. Nucleic Acids Res 36, W465-W469.

Eley, K. L., Halo, L. M., Song, Z. S., Powles, H., Cox, R. J., Bailey, A. M., Lazarus, C. M. \& Simpson, T. J. (2007). Biosynthesis of the 2-pyridone tenellin in the insect pathogenic fungus Beauveria bassiana. ChemBioChem 8, 289-297.

Figueiras, A. N. L., Girotti, J. R., Mijailovsky, S. J. \& Juarez, M. P. (2009). Epicuticular lipids induce aggregation in Chagas disease vectors. Parasit Vectors 2, 8.

Fujii, T., Nakamura, K., Shibuya, S., Tanase, S., Gotoh, O., Ogawa, T. \& Fukuda, H. (1997). Structural characterization of the gene and corresponding cDNA for the cytochrome P450rm from Rhodotorula minuta which catalyzes formation of isobutene and 4-hydroxylation of benzoate. Mol Gen Genet 256, 115-120.

Gotoh, O. (1993). Structure of P-450 genes in cytochrome P-450. In Cytochrome P-450, 2nd end, pp. 207-223. Edited by T. Omura, Y. Ishimura \& Y. Fujii-Kuriyama. Tokyo: Kodansha.

Graber, J. H., Cantor, C. R., Mohr, S. C. \& Smith, T. F. (1999). Genomic detection of new yeast pre-mRNA 3'-end-processing signals. Nucleic Acids Res 27, 888-894.

Guengerich, F. P. (2001). Common and uncommon cytochrome $\mathrm{P} 450$ reactions related to metabolism and chemical toxicity. Chem Res Toxicol 14, 611-650.

Halo, L. M., Heneghan, M. N., Yakasai, A. A., Song, Z., Williams, K., Bailey, A. M., Cox, R. J., Lazarus, C. M. \& Simpson, T. J. (2008). Late stage oxidations during the biosynthesis of the 2-pyridone tenellin in the entomopathogenic fungus Beauveria bassiana. J Am Chem Soc 130, 17988-17996.

Hamilton, R., Watanabe, C. K. \& Deboer, H. A. (1987). Compilation and comparison of the sequence context around the AUG start codons in Saccharomyces cerevisiae messenger-RNAs. Nucleic Acids Res 15, 3581-3593.

Holder, D. J. \& Keyhani, N. O. (2005). Adhesion of the entomopathogenic fungus Beauveria (Cordyceps) bassiana to substrata. Appl Environ Microbiol 71, 5260-5266.
Holder, D. J., Kirkland, B. H., Lewis, M. W. \& Keyhani, N. O. (2007). Surface characteristics of the entomopathogenic fungus Beauveria (Cordyceps) bassiana. Microbiology 153, 3448-3457.

lida, T., Ohta, A. \& Takagi, M. (1998). Cloning and characterization of an $n$-alkane-inducible cytochrome P450 gene essential for $n$-decane assimilation by Yarrowia lipolytica. Yeast 14, 1387-1397.

Juarez, P. (1994). Inhibition of cuticular lipid synthesis and its effect on insect survival. Arch Insect Biochem Physiol 25, 177-191.

Juarez, M. P. \& Fernandez, G. C. (2007). Cuticular hydrocarbons of triatomines. Comp Biochem Physiol A Mol Integr Physiol 147, 711730.

Lewis, M. W., Robalino, I. V. \& Keyhani, N. O. (2009). Uptake of the fluorescent probe FM4-64 by hyphae and haemolymph-derived in vivo hyphal bodies of the entomopathogenic fungus Beauveria bassiana. Microbiology 155, 3110-3120.

Lottermoser, K., Schunck, W. H. \& Asperger, O. (1996). Cytochromes P450 of the sophorose lipid-producing yeast Candida apicola: heterogeneity and polymerase chain reaction-mediated cloning of two genes. Yeast 12, 565-575.

Matsuzaki, F. \& Wariishi, H. (2005). Molecular characterization of cytochrome P450 catalyzing hydroxylation of benzoates from the white-rot fungus Phanerochaete chrysosporium. Biochem Biophys Res Commun 334, 1184-1190.

Napolitano, R. \& Juarez, M. P. (1997). Entomopathogenous fungi degrade epicuticular hydrocarbons of Triatoma infestans. Arch Biochem Biophys 344, 208-214.

Ohkuma, M., Muraoka, S., Tanimoto, T., Fujii, M., Ohta, A. \& Takagi, M. (1995). Cyp52 (Cytochrome-P450alk) multigene family in Candida maltosa - identification and characterization of 8 members. DNA Cell Biol 14, 163-173.

Ortiz de Montellano, P. R. (2005). Cytochrome P450: Structure, Mechanism, and Biochemistry, pp. 689. New York: Kluwer Academic/ Plenum.

Pedrini, N., Crespo, R. \& Juarez, M. P. (2007). Biochemistry of insect epicuticle degradation by entomopathogenic fungi. Comp Biochem Physiol C Toxicol Pharmacol 146, 124-137.

Pedrini, N., Mijailovsky, S. J., Girotti, J. R., Stariolo, R., Cardozo, R. M., Gentile, A. \& Juarez, M. P. (2009). Control of pyrethroid-resistant Chagas disease vectors with entomopathogenic fungi. PLoS Negl Trop Dis 3, e434.

Sanglard, D. \& Loper, J. C. (1989). Characterization of the alkaneinducible cytochrome-P450 (P450alk) gene from the yeast Candida tropicalis - identification of a new $\mathrm{P} 450$ gene family. Gene 76, 121136.

Scheller, U., Zimmer, T., Becher, D., Schauer, F. \& Schunck, W. H. (1998). Oxygenation cascade in conversion of $n$-alkanes to alpha, omega-dioic acids catalyzed by cytochrome p450 52A3. J Biol Chem 273, 32528-32534.

Seghezzi, W., Sanglard, D. \& Fiechter, A. (1991). Characterization of a 2nd alkane-inducible cytochrome-P450 encoding gene, Cyp52a2, from Candida tropicalis. Gene 106, 51-60.

Seghezzi, W., Meili, C., Ruffiner, R., Kuenzi, R., Sanglard, D. \& Fiechter, A. (1992). Identification and characterization of additional members of the cytochrome-P450 multigene family Cyp52 of Candida tropicalis. DNA Cell Biol 11, 767-780.

Shen, Y., Ji, G., Haas, B. J., Wu, X., Zheng, J., Reese, G. J. \& Li, O. Q. (2008). Genome level analysis of rice mRNA $3^{\prime}$-end processing signals and alternative polyadenylation. Nucleic Acids Res 36, 3150-3161.

Swanson, H. I. (2002). DNA binding and protein interactions of the AhR/ARNT heterodimer that facilitate gene activation. Chem Biol Interact 141, 63-76. 
Tanaka, A. \& Fukui, S. (1989). Metabolism of $n$-alkanes. In The Yeast, pp. 261-287. Edited by A. Tanaka \& S. Fukui. New York: Academic Press.

Tanaka, A. \& Ueda, M. (1993). Assimilation of alkanes by yeasts: functions and biogenesis of peroxisomes. Mycol Res 97, 1025-1044.

Treger, J. M., Magee, T. R. \& McEntee, K. (1998). Functional analysis of the stress response element and its role in the multistress response of Saccharomyces cerevisiae. Biochem Biophys Res Commun 243, 13-19.

Tsuchiya, Y., Nakajima, M. \& Yokoi, T. (2003). Critical enhancer region to which AhR/ARNT and Sp1 bind in the human CYP1B1 gene. J Biochem 133, 583-592.

van Beilen, J. B., Li, Z., Duetz, W. A., Smits, T. H. M. \& Witholt, B. (2003). Diversity of alkane hydroxylase systems in the environment. Oil \& Gas Sci Technol - Rev IFP 58, 427-440.
Wanchoo, A., Lewis, M. W. \& Keyhani, N. O. (2009). Lectin mapping reveals stage-specific display of surface carbohydrates in in vitro and haemolymph-derived cells of the entomopathogenic fungus Beauveria bassiana. Microbiology 155, 3121-3133.

Yadav, J. S. \& Loper, J. C. (1999). Multiple P450alk (cytochrome P450 alkane hydroxylase) genes from the halotolerant yeast Debaryomyces hansenii. Gene 226, 139-146.

Yadav, J. S., Soellner, M. B., Loper, J. C. \& Mishra, P. K. (2003). Tandem cytochrome P450 monooxygenase genes and splice variants in the white rot fungus Phanerochaete chrysosporium: cloning, sequence analysis, and regulation of differential expression. Fungal Genet Biol 38, 10-21.

Edited by: B. A. Horwitz 